\title{
VISUALISATION SUPPORT FOR EXPLORING URBAN SPACE AND PLACE
}

\author{
C. Pettit a, * I. Widjaja b, P. Russoa, R. Sinnott b, R. Stimson a, M. Tomko a \\ a Australian Urban Research Infrastructure Network, Faculty of Architecture, Building and Planning, The University of \\ Melbourne, 3010 VIC, Australia \\ ь Melbourne eResearch Group, Faculty of Engineering, The University of Melbourne, 3010 VIC, Australia \\ (cpettit, ivow, patrizia.russo, rsinnott, rstimson, tomkom)@unimelb.edu.au
}

\section{Commission VI, WG VI/4}

KEY WORDS: Geo-visualisation, e-Infrastructure, Visual Analytics, Dashboards, Urban Research

\begin{abstract}
:
Urban research is fundamentally underpinned by heterogeneous, highly varied data. The availability and quantity of digital data sources is increasing rapidly. In order to facilitate decision-making and support processes related to urban policy and management, such data has to be readily analysed, synthesised and the results readily communicated to support evidence based decision-making. In this paper, we consider the current state of play of visualisation as it supports urban research. In doing so we firstly consider visualisation environments such as geographical information systems (GIS) and Cartography tools, digital globes, virtual simulation environments, building information models and gaming platforms. Secondly, we consider a number of visualisation techniques with a focusing on GIS and Cartography tools including space time cubes, heat maps, choropleth maps, flow maps and brushing.
\end{abstract}

This review of visualisation environments and techniques is undertaken in the context of the Australian Urban Research Infrastructure Network project (www.aurin.org.au). AURIN is tasked with developing a portal and associated e-Infrastructure, which provides seamless access to federated data, modelling and visualisation tools to support the urban researcher community in Australia. We conclude by outlining future research and development opportunities in developing the AURIN visualisation toolkit by reflecting on the value of visualisation as a data exploration and communication tool for researchers and decision-makers to assist with the study and management of the urban fabric.

\section{INTRODUCTION}

In this paper we survey a range of visualisation tools and techniques that can support the fundamental information needs of researchers and practitioners concerned with issues of the built environment. Our approach is a pragmatic one, stemming from the need to build an online "lab in a portal" environment, providing access to a broad range of spatial and temporal datasets to support urban research and decision-making in Australia. The Australian Urban Research Infrastructure Network (AURIN) is an e-Infrastructure initiative tasked with developing open source and open standard online data access and interrogation tools to facilitate world class urban research (Sinnott et al., 2011).

By their very nature because most urban problems are inherently spatial hence this paper will focus primarily but not exclusively on geo-visualisation tools and techniques. In this review paper we are interested in visualisation environments that can support a broad range of urban research domains (Lenses), ranging from issues of economics and productivity, population health, housing, transport, risk and vulnerability, to water and energy consumption, logistics and urban design. These areas have natural synergies and overlaps, and thus complementary visualisation environments and techniques are applicable across a number of them.

\section{VISUALISATION FOR URBAN RESEARCH}

At the beginning of 21 st century, about half of the world's population lived in urban areas (Gottdiener and Hutchison, 2010). The United Nations (2003) anticipated that in 2015 the world would have 358 urban agglomerations with a population exceeding 10 million. While two of Australia's biggest metropolitan areas, Sydney and Melbourne, are still far from being considered mega-cities, urbanisation is one process changing the face of Australian cities (Randolph, 2004). Understanding the processes that underpin the function and changing nature of urban space and place is complex and can benefit from modelling, simulation and visualisation. It is the later of these which forms the focus of this paper in the context of space (the geometric construct) and place (the dimension of lived experience).

Visualisation environments and techniques provide an important function in communicating urban research and support collaborative endeavours. Advances in digital technologies and the wide availability of the Internet enables the production, manipulation, and sharing of vast digital data resources many of which contain geospatial references (Andrienko et al., 2007). The use of mobile devices and social network platforms such as Twitter has given birth to novel forms of geospatial data collection. As part of e-government initiatives, public agencies at various levels have begun to publish their data online under the auspices of Government 2.0 and public sector release frameworks (Ding et al., 2010). For example, in Australia the Australian Bureau of Statistics (ABS) provides access to a broad range of socio-economic datasets. With the advent of more digital data becoming increasingly available there are novel ways urban researchers can now explore urban space and place in novel ways, supported by a wide array of visualisation tools and techniques.

Advances in technology and data infrastructure provide the backdrop for unique opportunities to the urban researcher and decision-maker in trying to make better sense of growing deluge 
of digital, predominantly geospatial data. Visualisation has been identified as a way to unearth insights and patterns from this data deluge; this often comes under the umbrella term of visual analytics (Keim et al., 2008a). Visualisation, combined with interaction design and data analysis, can provide a way for the researcher to maximise their cognitive ability to deal with the dimensionality and complexity of data.

Geospatial data poses additional challenges to available visualisation approaches. Andrienko et al. (2007) identified three distinctive aspects of geovisual analytics: (i) the complexity of geospatial space, (ii) multiple roles, and (iii) tacit criteria and knowledge. These challenges are acutely apparent in the context of large multidisciplinary e-infrastructure projects such AURIN. The need to provide data interrogation tools across to a broad number of urban research communities, planners and policy-makers requires an understanding of what tool best suits a particularly user group.

The complexity of space (Andrienko et al., 2007) is an issue in analysing data related to geospatial space (regions) and their boundaries defined based on divergent criteria. In a multidisciplinary data playground as being developed by einfrastructure initiatives such as AURIN, there is a need to integrate data relating to defined geographies (administrative regions, political boundaries), less vague and vernacular regions and 'places', and ad-hoc, purpose-made areas of interest defined by researchers. These different geographic spaces (also known as regionalisations), not only introduce an enumeration of places but often also their hierarchy evolving in time.

The challenge posed by multiple user roles is critical in the context of AURIN, with diverse users and stakeholders who wish to explore and analyse Australian urban space and place. One of the challenges facing AURIN is the need to support not only various types of urban researchers, but also decisionmakers. With reference to the MacEachren geovisualisation cube, (MacEachren et al., 2004) (Fig. 1), which helps us understand the dimensions of visualisation and communication, we illustrate how researchers and decision-makers differ in terms of interface interaction level, task type and geovisualisation function. Focusing on the Users axis we expect the urban researcher with a deep knowledge to be more of a specialist while we place the decision-maker with a more broad knowledge near the middle of the axis. This suggests the researcher may wish to utilise high-interactive interfaces in order to explore multi-dimensional data and decision spaces. The decision-maker, in contrast, is likely to prefer a lower interactive environment and analyses and synthesises the gained information for supporting real world decision-making. Owing to these differing user needs a toolkit with a rich set of data interrogation techniques and decision support functionality will likely be required in the AURIN portal and will subsequently be discussed in this paper.

The heterogeneity of users is further compounded by the multidisciplinary array of end users. AURIN has proceeded with 10 strategic implementation streams-called Lenses (see http://aurin.org.au). These lenses provide a construct from which to explore and research the urban fabric.

AURIN needs to facilitate the analysis of geospatial data not only by domain experts in each given Lens, but also to support interdisciplinary scientific inquiry. The role of visualisation in, and across each of these Lenses could be of presentation, confirmatory, exploratory uses, or their combination (Keim et al., 2008b). A further challenge for AURIN is to capture the tacit knowledge contained within the urban research community and provide a way for the users to bring in and share that knowledge, in particular across disciplinary boundaries.

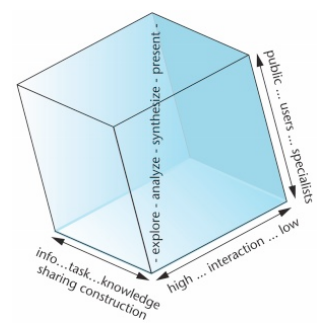

Figure 1. MacEachren's (2004) geovisualisation cube with key dimensions relating to human and map interaction (MacEachren et al., 2004).

\section{CURRENT STATE OF PLAY OF VISUALISATION SUPPORTING URBAN RESEARCH}

Approaching visualisation in a large multidisciplinary initiative such as AURIN requires a pragmatic yet somewhat critical review of the ways visualisation can be used to represent and to analyse data. We carry out this review using a dual approach. Firstly, we investigate some of the technology environments where visualisation can take place. These environments provide the playground and context for the interaction between the user, data, and the visualisation product. Researchers from a specific discipline may be familiar with a particular type of technology environment but not others. For example, urban sociologists might be more comfortable driving visualisation from statistical tools like R or SPSS. However, urban planners would likely be more conversant with the visualisation capability within GIS and Planning Support System (PSS) tools. This review assists us to broadly consider the possible interaction paradigms to be supported by the visualisation tools that we are going to develop.

Secondly, we look at some techniques that have been traditionally used to visualise geospatial data. This is not intended as an exhaustive review of all the relevant visualisation techniques. Instead, we are looking to get a sense of complexity in maintaining support for various typical visualisation techniques and to aim for flexibility in including more novel techniques.

\subsection{Visualisation environments}

A visualisation environment establishes a metaphor and context for the interacting of data and its various visual form. It provides a place for the user to select, process, present, and interact with data accessible via various visualisation approaches. In this paper we discuss several types of visualisation environments that are widely associated with the exploration and analysis of geospatial data representing the built environment. This list provides a non-exhaustive pragmatic overview of the environments of relevance for AURIN.

GIS and Cartography Tools: The powerful interactive data interrogation capabilities of GIS are well recognised by the urban research community (Claramunt et al., 2000). GIS has been successfully used to support urban practitioners in decision-making (Kodmany, 2001). With the ability to overlay 


\begin{tabular}{lllll}
\hline Technique & $\begin{array}{l}\text { Static/ } \\
\text { Dynamic }\end{array}$ & Dimensionality & Geographical Scale & Scenarios of usage \\
\hline Space Time Cube & Dynamic & 3D & $\begin{array}{l}\text { State, Local Govt (LGA), Suburb (SLA), } \\
\text { Census Districts (CD) }\end{array}$ & Display of movement \\
\hline Heat map & $\begin{array}{l}\text { Mostly static, but can be animated over } \\
\text { time }\end{array}$ & $\begin{array}{l}\text { 2D Points (and optionally continuous } \\
\text { measure) }\end{array}$ & State, LGA, SLA, CDs & Land use change \\
\hline Choropleth map & $\begin{array}{l}\text { Mostly static, but can be animated over } \\
\text { time }\end{array}$ & 2D Area and a continuous measure & State, LGA, SLA, CDs & $\begin{array}{l}\text { Snapshot of socio-economic indicators for } \\
\text { administrative areas }\end{array}$ \\
\hline Flow Map & Static/Dynamic & Graph (2D Points and Vectors) & State & $\begin{array}{l}\text { Inspect migration across states, individual } \\
\text { population movement }\end{array}$ \\
\hline Brushing & Dynamic & N/A & $\begin{array}{l}\text { Individual to broad scale (State) (point and } \\
\text { polygon) }\end{array}$ & $\begin{array}{l}\text { Exploring detailed socio-economic data } \\
\text { variables within a given aggregated } \\
\text { geographhy }\end{array}$ \\
\hline Graph \& Charts & Static & $\begin{array}{l}\text { Ordinal/nominal measures and some } \\
\text { continuous measures. }\end{array}$ & State, LGA, SLA, CDs & $\begin{array}{l}\text { Presenting a snapshot of socio-economic } \\
\text { indicators in a dashboard }\end{array}$ \\
\hline Table 1. Summary of visualisation techniques relevant to AURIN projects &
\end{tabular}

Table 1. Summary of visualisation techniques relevant to AURIN projects

data sets, filter them spatially, and undertake a range of advanced geographical analyse, GIS facilitates the comparison of scenarios and supports urban management (Köninger and Bartel, 1997).Understanding the urban space and place requires contributions from many disciplines including: geography, demography, urban design, transport planning, population health etc... Furthermore, much data that both describes and can be used to model urban space and place is heterogeneous. Therefore, intuitive and easily manageable interfaces, which provide access to a discrete set of GIS functionalities required by specific user groups are highly desirable (Lanter and Essinger, 1991).

Digital Globes: Digital globes such as Google Earth, NASA WorldWind, ArcGIS Explorer allow the user to zoom in, flythrough and walk-through many cities and urban spaces comprising our planet. These digital viewers can typically support two and half dimensional (2.5D) exploration of photorealistically real-world structures and phenomena, supporting a visual understanding of geospatial relations in the environment (Smith, 2007). The ease of use in navigating these digital globes is a significant factor why they are encountering vast popularity in use by urban researchers, decision-makers and community groups in exploring urban planning and environmental management decision spaces (Sheppard and Cizek, 2008). For those urban researchers not very familiar with GIS, digital globe interfaces provide an intuitive visualisation environment for data exploration. Google Earth, for example, is primarily directed to non GIS-users (Smith, 2007). Moreover, multiple institutions and organisations are sharing and communicating their data through the open standard KML data format, which is supported by most digital globe products. Hence, digital globes are considered a valuable tool facilitating collaboration and decision-making (Aurambout and Pettit, 2008).

Virtual Simulation Environments: Virtual simulation environments (VSE) can represent urban landscape in three dimensions. Physical and environmental objects (buildings, vegetation, etc.) and also dynamic phenomena such as traffic, crowds and weather can be photo-realistically displayed, and be attributed behaviours (Kodmany, 2001). As a result, VSE are increasingly used by urban planners and architects (Hamill and O'Sullivan, 2003). VSE allow users to model urban environments by providing a wide range of operations such as the removal, insertion and motion of $3 \mathrm{D}$ elements (Drettakis et al., 2006). GIS data and associated computer simulation models can typically either be imported or programmed directly into a VSE (Batty, 1994). This enables users to explore past, present and future urban landscapes. By means of VSE urban researchers may simulate real-world aspects and test different scenarios (Drettakis et al., 2006). Examples of such environments include UrbanCircus, (http://www.circus3d.com/) and UrbanSim (www.urbansim.org).

Building Information Models: A Building information model (BIM) enables the representation of detailed building models, including relations and attributes of the detailed elements of buildings. The capability of a BIM to include form, material and technical characteristics of buildings make it an appealing communication and design tool for architects, engineers and building managers/designers (Schlueter and Thesseling, 2009). Moreover, BIMs typically support detailed 3D object interrogation such as measurement and attribute queries (e.g. building material specifications), which allows users to better understand building performance and design issues. In this way performance and design assessments may be undertaken through exploration of an interactive 3D BIM. The use of BIMs as an urban design and planning support tool has been gradually increasing in the building industry (Schlueter and Thesseling, 2009). However, there are a limited number of tools available to support the integration of BIMs with contextual space and place information (for example, Autodesk's Revit and Tekla's Structures BIM software packages).

Game Platforms: Contemporary game platforms (or game engines) such as Unity3D, FarCry, Virtools offer performance and high quality visual presentation of urban space and place. Gaming environments also offer complex interactivity, and high portability through pervasive platform support (including mobile devices). These environments provide a different level of visualisation sophistication (Fritsch and Kada, 2004). Another feature that is rarely available in other visualisation environments is the support for connectivity through networking or multi-user feature. While VSE bring realism to the built environment, game environments add in interactivity to the experience. Game environments can thereby facilitate social interaction and collaboration between users within a virtual space. This elevates the basic function of visualisation for communication purposes, to support collaborative design process, parallelism, and multi-tasking activities (Indraprastha and Shinozaki, 2009). In an urban context, game environments have been used to explore human way-finding and navigation abilities within the built environment (Germanchis et. al., 2005).

\subsection{Visualisation Techniques}

Other than traditional visualisation techniques like graphs and charts (bar chart, scatterplot, line chart), AURIN aims to 
provide a set of contemporary geo-visualisation techniques made accessible via a portal interface. This section discusses some of these techniques. Each technique is classified in terms of key criteria relevant to understanding urban space and place (see Table 1).

Space Time Cube: An open source application of the Space Time Cube (STC) exists within the GIS uDig desktop software (User-friendly Desktop Internet GIS). uDig provides a broad range of functions and interactivity tools in relation to the STC application. It allows the use to view the cube with its content from any direction and at different scales as well as to modify data appearance. The visualisation of so-called Space Time Paths (STP) in the STC, allows one to depict movements of any object or subject through the space in two dimensions and through time, which is depicted as the third dimension (Li et al., 2010). In this way the STC representation conveys the dynamics of movement trajectories. To provider greater geospatial context into account, it is possible to place a map or a digital terrain model as a data layer on the bottom of the STC. A further function of uDig's STC application is the opportunity to link additional data to the STP. A rich set of multimedia data including video clips, photos and text) can be linked with branches to the STP. These so-called annotations are substituted by symbol objects (icons, geometric symbols) in the STC. By clicking on the symbol object, the annotation is displayed. The aim of so-called annotated STP (ASTP) is to complement the visualisation with contextual material. Such a visualisation technique that includes spatial, temporal and thematic dimensions may support urban research in for example, understanding household travel behaviour.

Heat Map (also known as density surfaces): In a geospatial context, a heat map is a visualisation technique for representing the density of spatial data, using Kernel Density Estimation. Heat maps enable viewers to quickly identify high density areas without losing the general spatial context. This is typically useful when analysing multivariate geospatial data, where users need to get a sense of the correlation between geographical features and another measurement. Heat maps can provide an overview of distribution of a variable as well as highlight local 'hotspots'. In the context of urban studies, heat maps can be useful to identify correlation and characterise distribution within socio-spatial data. For example, (Bak et al., 2010) use heat maps to visualise the centrality of land uses in the city of Raanana, Israel. Heat maps are most useful in also mapping temporal urban phenomena such as people and traffic flows (Ihaddadene and Djerba, 2008).

Choropleth Maps: Choropleth maps represent aggregated measures of pre-defined regions with crisp boundaries (Wright, 1938). Choropleth mapping is particularly useful in providing comparative summaries over specified geographies. In the context of urban studies, choropleth mapping is widely used to broadly compare administrative regions based on given characteristics, such as socio-spatial indicators. For example, (Chhetri et al., 2011) use choropleth maps to visualise circular dispersion of the journey to work patterns across city of Brisbane in Australia.

Flow Map: Flow maps display movements of objects or subjects from one place to another by means of lines or arrows. The data used therefore has different initial and end geographical locations within the analysed time period such as, for example, migration patterns between regions.The lines/arrows on the map vary in width to depict the number of objects or subjects that have changed their location (Phan et al., 2005). Flow maps are typically static, however can be made dynamic through deploying time sequence animation.

Brushing (also known as multiple-linked views): A method for dynamic querying by direct manipulation of visual and data displays with the results being updated based on manipulation is commonly referred to as "brushing" . Brushing is typically used for exploratory data analysis (Andrienko and Andrienko, 2006). In brushing, multiple views (data table or visualisations) exist and selection of a particular feature/attribute in one view would provide a relevant display in other views. Examples for brushing in a geospatial area can be seen in GeoDa (www.oicweave.org).

\section{TOWARDS A VISUALISATION TOOLKIT}

In designing the AURIN visualisation toolkit and its enabling online technology platform (known as the AURIN portal) we need to consider functionality from an end users' perspective. To adequately support end users consideration needs to be given to the functional requirements of presentation, interaction, exploration and explanation (Andrienko et al., 2010).

\subsection{Visualisation Users}

The AURIN project aims to provide visualisation tools for two different end user groups (see also section 2): (i) urban researchers by providing geo-visual analytics; and (ii) decisionmakers by providing information via the metaphor of the dashboard. This circumstance leads to diverse scenarios of usage.

\subsection{Visual Analytics for Urban Researchers}

Visual analytics can be described as "the science of analytical reasoning facilitated by interactive visual interfaces" (Thomas and Cook, 2005). The goal of visual analytics is not only to visually represent the information, but also to provide an iterative mechanism to allow the user to directly interact with the information and its representations, to analyse and to obtain new insights from the original information. Visual analytics aims to reduce information complexity (multidimensionality) by employing data analytics or data mining tools and human superiority in doing visual cognitive task. The goal of visual analytics research is to turn the data deluge into knowledge (Keim et al., 2008a).

In the AURIN context, visual analytics presents as cycles of interact-process-visualise-analyse. Interact involves data and representation exploration via zooming, filtering, focusing over spatial and temporal features. Process involves transformation into a better representation via mechanism such as simple aggregate statistic to feature extraction (for example, PCA). This could mean a derivation of a model for data like self -organising maps (SOMs) (Koua and Kraak, 2004), decision trees, or association rules. Visualise involves provision of visual representations from basic chartings to more abstract representation like a TreeMap (Slingsby et al., 2010). Initially the AURIN portal has supported data visualisation via thematic maps and bar graphs. There is work underway in being able to support the visualisation of large, crowd-sourced georeferenced datasets, including their temporal dynamics, in conjunction with traditional authoritative datasets. 


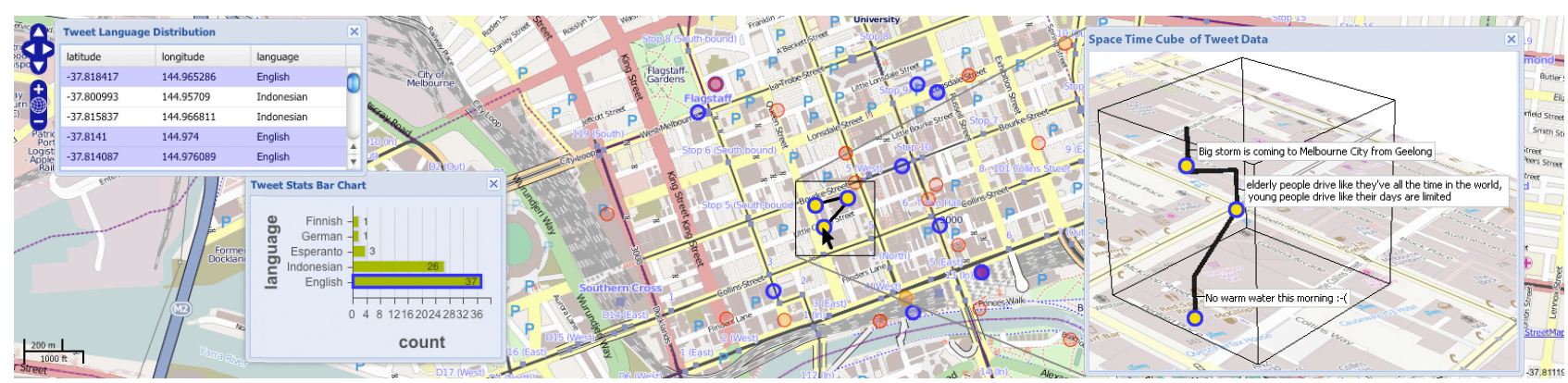

Figure 2. An example of tweets with the Melbourne Central Business District (CBD) dynamically visualised via linked maps, graphs and a Space Time Cube, where multiple views can be dynamically interrogated through a GIS and Cartographic brushing technique.

Analyse involves the provision of tools to enable visual data mining of new relationships, brushing can somewhat support this requirement. Figure 2 demonstrates the use of Twitter data streams from around Melbourne CBD, where a brushing technique is enabled to support the exploration of different views of the same dataset. A user can selectively inspect a slice of data defined by an attribute (for example, a language), and explore the spatial and temporal dynamics of records in this language, and compare across languages. Furthermore, these tweets which have a geo-location can be visually interrogated via a STC. Further interrogation of the data would enable users to hotlink from annotated data within the STC to further associated textual, imagery and video footage.

\subsection{Dashboard for Decision-makers}

Government policy-makers and planners are increasingly requesting an evidenced based approach to decision making. This should support both real-time and operational decisions. For example, responding to an emergency situation such as a fire or food, or for making strategic long term decisions in planning for the growth of cities, or preparing for a future impacted by a changing climate.

Dashboards comprising maps and indicator dials which link to either or both real-time data and computer simulation data are seen as way of connecting policy-makers and planners with information they need to deal with both operational and strategic decisions. Dashboards have been taken up by the geographical sciences community as a form of visual interface to enable an evidence based approach to decision-making. Figure 3 provides an example of a dashboard for decisionmakers focusing on water resource management. The dashboard includes a Google Earth viewer to present geographical information simultaneously with indicators integrating pie charts, graphs and tables to convey thematic information.

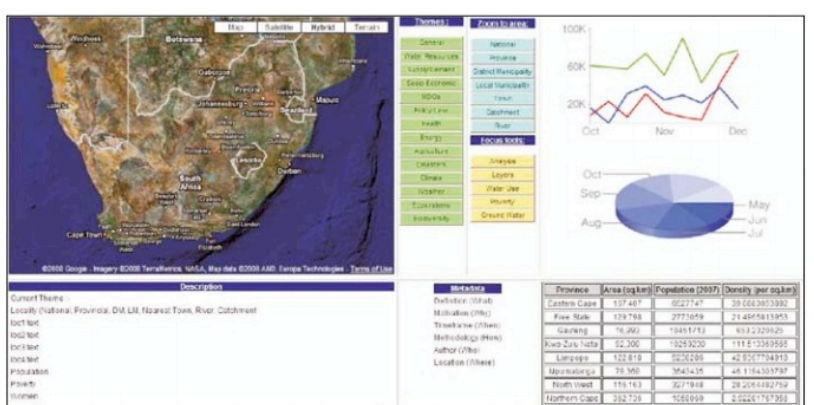

Figure 3. IWRDSS Dashboard (Integrated Water Resource Decision Support System) with Google Earth map, pie chart, graph and table (Craigie, 2008).
Decision-makers need not understand all the computation algorithms and workflows required to create a computer simulation model. However, if they trust the underlying assumptions and models then they can interact with a series of simple mapping and indicator interfaces which can provide them the information to make real world decisions. In designing dashboard this should be done in conjunction with the end users to ensure that what is displayed is what the decision-maker requires (useful) and is in a form that is easy to user (usability). Therefore, principles of usability should be followed in designing such a dashboard (Koua et al., 2006).

\section{FUTURE RESEARCH AND DEVELOPMENT}

Future research and development of the visualisation capabilities within the AURIN project will progressively see the development of a more sophisticated visualisation toolkit. Such a toolkit will provide a rich set of data interrogation techniques and decision support functionality, as discussed in this paper. It is anticipated visual analytic techniques which utilise eyetracking software will also be deployed. This will assist in understanding and improving the usability of e-research data exploration and decision support tools as they are progressively developed and made accessible via the AURIN portal to support urban researchers and decision-makers across Australia.

\section{References}

Andrienko, N., Andrienko, G., 2006. Exploratory analysis of spatial and temporal data: a systematic approach. Springer, Berlin.

Andrienko, G., Andrienko, N., Jankowski, P., Keim, D., Kraak, M. -J., MacEachren, A., Wrobel, S., 2007. Geovisualization analytics for spatial decision support: setting the research agenda. International Journal of Geographical Information Science, 21(8), pp. 839-857.

Andrienko, G., Andrienko, N., Demsar, U., Dransch, D., Dykes, J., Fabrikant, S. I., Jern, M., Kraak, M.-J., Schumann, H., Tominski, C., 2010. Space, time and visual analytics. International Journal of Geographical Information Science, 24(10), pp. 1577-1600.

Aurambout, J.-P., Pettit, C., 2008. Digital globes: gates to the digital earth. Digital Earth Summit on Geoinformatics 2008.

Batty, M., 1994. Using GIS for visual simulation modeling. GIS World, 7(10), pp. 46-48. 
Bak, P., Omer, I., Schreck, T., 2010. Visual analytics of urban environments using high-resolution geographic data. In: Geospatial Thinking, Edit.: M. Painho, M. Santos, H. Pundt, Springer, Berlin, pp. 25-42.

Chhetri, P., Corcoran, J., Stimson, R., 2011. Using circular statistics to explore the geography of the journey to work. Papers in Regional Science, 88(1), pp. 119-132.

Claramunt, C., Jiang, B., Bargiela, A., 2000. A new framework for the integration, analysis and visualisation of urban traffic data within geographic information systems. Transportation Research, 8(1-6), pp. 167-184.

Craigie, D., 2008. Information integration: A GIS perspective, Ecological Circuits, pp. 15-19.

Ding, L., DiFranzo, D., Graves, A., Michaelis, J., Li, X., Guinness, D., Hendler, J., 2010. Data-gov wiki: towards linking government data. AAAI Spring Symposium on Linked Data Meets Artificial Intelligence, pp. 38-43.

Drettakis, G. , Roussou, M., Reche, A., Tsingos, N., 2006. Design and evaluation of a real-world virtual environment for architecture and urban planning. Presence Teleoperators and Virtual Environm., 16(3), pp. 1-21.

Fritsch, D., Kada, M., 2004. Visualisation using game engines. Archivum ISPRS, 35.

Germanchis, T., Cartwright, W., Pettit, C., 2005. Using computer gaming technology to explore human wayfinding and navigation abilities within the built environment. Proc. XXII International Cartographic Conference.

Gottdiener, M., Hutchison, R., 2010. The new urban sociology, fourth edition, Westview Press, Boulder, USA.

Hamill, J., O'Sullivan, C., 2003. Virtual Dublin - a framework for real-time urban. Simulation Journal of WSCG, pp. 1-5.

Ihaddadene, N., Djeraba, C., 2008. Real-time crowd motion analysis. Pattern Recognition ICPR 2008, pp. 1-4.

Indraprastha, A., Shinozaki, M., 2009. The investigation on using Unity3D game engine in urban design study. ITB Journal of Information and Communication Technology, 3(1), pp. 1-18.

Keim, D., Andrienko, G., Fekete, J. D., Görg, C., Kohlhammer, J., Melançon, G., 2008a. Visual analytics: definition, process, and challenges. In: Information Visualization, Edit.: A. Kerren, J. Stasko, J.-D. Fekete, C. North, Springer, Berlin, pp. 154-175.

Keim, D., Mansmann, F., Schneidewind, J., Thomas, J., Ziegler, H., 2008b. Visual analytics: scope and challenges. In: Visual Data Mining, Edit.: S. Simoff, M. Böhlen, A. Mazeika, Springer, Berlin, pp. 76-90.

Kodmany, K, 2001. Visualization tools and methods. for participatory planning and design. Journal of Urban Techn., pp. $1-37$.

Koua, E., MacEachren, A., Kraak, M.J., 2006. Evaluating the usability of visualisation methods in an exploratory geovisualisation environment. International Journal of
Geographical Information Science, 20(4), pp. 425-448.

Koua, E., Kraak, M. J., 2004. Alternative visualization of large geospatial datasets. Cartographic Journal, 41(3), pp. 217-228.

Köninger, A., Bartel, S., 1997. 3D-GIS for urban purposes. GeoInformatica, pp. 79-103.

Lanter, D., Essinger, R., 1991. User-centered graphical user interface design for GIS, pp. 1-24.

Li, X., Coltekin, A., Kraak, M-J., 2010. Visual exploration of eye movement data using the space time cube. In: Geographic Information Science, Edit.: S. I. Fabrikant et al., Springer, Berlin, pp. 295-309.

MacEachren, A., Gahegan, M., Pike, W., Brewer, I., Cai, G., Lengerich, E., Hardisty, F., 2004. Geovisualisation for knowledge construction and decision support. IEEE Comput Graph Applications, 24(1), pp. 13-17.

Phan, D., Xiao, L., Yeh, R., Hanrahan, P., 2005. Flow map layout. Information Visualisation, pp. 219-224.

Randolph, B., 2004. The changing Australian city: new patterns, new policies and new research needs1. Urban Policy and Research, 22(4), pp. 481-493.

Schlueter, A., Thesseling F., 2009. Building information model based energy/exergy performance assessment in early design stages. Automation in Construction, 18, pp. 153-163.

Sheppard, S. R. J., Cizek, P., 2008. The ethics of google earth: crossing thresholds from spatial data to landscape visualisation. Journal of Environment Management, 90, pp. 2102-2117.

Sinnott, R. O., Galang, G.,Tomko, M., Stimson, R., 2011. Towards an e-Infrastructure for Urban Research across Australia. IEEE $7^{\text {th }}$ International Conference on E-Science, Stockholm, Sweden, pp. $295-302$.

Slingsby, A., Wood, J., Dykes, J., 2010. Treemap cartography for showing spatial and temporal traffic patterns. Journal of Maps, pp. 135-146.

Smith, A., 2007. Digital urban - the visual city. Working Papers Series. Paper 124. Centre for Advanced Spatial Analysis, University College London.

United Nations, 2003. World urbanisation prospects: the 2003 revision.http://www.un.org/esa/population/publications/wup200 3/WUP2003Report.pdf, Access: 18.4.2012.

Thomas, J., Cook, K. A. (2005). Illuminating the path: The research and development agenda for visual analytics, IEEE computer graphics and applications, 26 (1), pp. 10-13.

Wright, J.K., 1938. Problems in population mapping. Notes on statistical mapping, with special reference to the mapping of population phenomenon, pp. 1-18. 